\title{
AGRESIVIDAD EN NIÑOS Y NIÑAS DE KINDER Y PRIMER CICLO, DEL ÁREA METROPOLITANA
}

\section{AGGRESSIVENESS IN CHILDREN OF KINDER AND ELEMENTARY SCHOOL OF METROPOLITAN AREA}

\section{Viriam Leiva Díaz*}

\begin{abstract}
RESUMEN
Este estudio es una investigación de corte descriptivo con un total de 154 escolares, en cuatro escuelas del área metropolitana, Costa Rica, en el 2005. Se definieron cuatro variables relacionados con la agresión para analizar: datos sociodemográficos, conocimiento de los conceptos de agresión en el área escolar y familiar. Los resultados indican que, tanto en la escuela como en sus casas, ellos y ellas refieren actos violentos de magnitud variable.
\end{abstract}

PALABRAS CLAVES: COSTA RICA * NIÑEZ * AGRESIÓN * ESCUELA * FAMILIA

ABSTRACT

In this descriptive investigation, including 154 children between 5 and 9 years old, in four different schools from the big metropolitan area. Four variables were defined: socio demographic data, aggressive knowledge concepts, scholar and familial areas in relation with aggression. The results show that at school and house, children perceive aggressive behaviors of different magnitude, and identify the role-played in the dynamics of aggression.

KEY WORDS: COSTA RICA * CHILDREN * AGGRESSION * SCHOOL * FAMILY

\section{INTRODUCCIÓN}

La agresión se ha instalado en nuestras vidas. La hemos asumido como una manera de ser $y$ de estar en el mundo; la intolerancia a la diferencia, la exclusión, la ausencia del diálogo, la resolución de conflicto por medio de la agresión, todos se han vuelto comportamientos cotidianos.

Cátedra de Psicopedagogía, Universidad de Costa Rica viriaml@yahoo.es 
Hay una dicotomía en nuestros valores: bueno o malo, no hay espacios para matices en el caleidoscopio social; no existe la pluralidad. El otro ser social es un potencial enemigo, del cual hay que estar a la defensiva, $y$ a la ofensiva, aun en los ambientes más cercanos, como la familia, la escuela, el trabajo. La palabra ha perdido su poder para ser una herramienta eficaz para solventar las diferencias; se desconoce al otro social como interlocutor legítimo, se le niega su condición de ser humano.

Todas las instituciones en nuestra sociedad, llámese familia, escuela, iglesia, entre otras, tienen su cuota en la forma en que están siendo socializados nuestros niños y niñas.

Como lo apunta Vigotsky (1978) la vivencia institucional empieza en el hogar, allí los niños $y$ niñas aprenden a vivir $y$ a con-vivir, donde se establecen las normas como mediadoras de los conflictos. Se aprende a participar, a tomar decisiones, a ejercer nuestros deberes como parte de una colectividad; es en este ámbito en donde se establecen una jerarquía de valores, según la cual actuamos, como ciudadanos y como personas. Luego, en la socialización secundaria, en la escuela, que es la segunda instancia mediatizadora en la formación de valores y normas que guían la convivencia entre las personas. Y no por último, sino tal vez más importante porque permea las instancias anteriores, los medios de comunicación a través principalmente de la televisión. Estas instancias requieren de padres, maestros, comunicadores reflexivos, concientes de su papel, que aceptan la diferencia, para que logren verdaderamente, enseñar a través del modelaje y así establecer una verdadera relación dialógica con las otras personas para resolver los conflictos. Sin embargo, la realidad es que la agresión, la intolerancia, permea todas las instancias antes mencionadas. Así la familia, se constituye en un lugar de desencuentro, inseguridad e inestabilidad social. Para esto, basta con abrir los periódicos cada mañana o revisar las estadísticas nacionales sobre femicidio, agresión intra familiar, niños $y$ niñas en la calle $y$ de la calle. De igual forma en las escuelas, las situaciones de agresión van en aumento, tenencia de armas por parte de los estudiantes, impotencia de los maestros, que se encuentran sin medios represivos, como es la expulsión del ámbito escolar; presencia de la policía, como medio represivo social, entre otros. Y por último, los medios de comunicación que exaltan situaciones de agresión, como una forma de mantener "rating", y ser el más leído, visto u oído, en una visión mercantilizada $y$ no mediatizada por la razón. Se exhiben programas con contenido violento y se le cede a los padres la responsabilidad de custodiar lo que ven los niños y niñas, descargando de esta manera su responsabilidad en la construcción de una jerarquía de valores que guíen un actuar ciudadano reflexivo, capaz de solucionar conflictos de una manera positiva.

Es importante conceptualizar la palabra agresión, ya que nos enfrentamos a una impresión lingüística en la que no se le da a la palabra agresión, la misma connotación semántica, ni la importancia social o legal, a situaciones que son agresivas, pero no catalogadas como tal, entre estas los insultos $y$ otras faltas llamadas "leves" a la disciplina. En contraste con aquellas a las que la opinión pública da la connotación de agresión como es el vandalismo o la agresión física, con secuelas de importancia, sean estas incapacitantes o mortales para la víctima.

Según The Word Health Organization (WHO) (Krug, E., 2002) la agresión es clasificada como interpersonal, intrapersonal y colectiva. El tipo interpersonal entre un oponente $y$ otro que puede ser un extraño, conocido, miembro de la familia o un compañero o compañera íntima. Aquí se incluyen actos tales como homicidio, violación y asalto agravado. La forma intrapersonal, se refiere a la agresión hacia uno mismo, que incluye el suicidio y los intentos de autoeliminación o gestos suicidas. La forma colectiva, hace referencia a la agresión social, política y económica concomitante entre grupos de personas, por el gobierno o por las instituciones, esta incluye entre otros, la agresión de pandillas, el abuso de los derechos humanos, o los actos económicos disruptivos intencionales contra muchos para beneficiar a unos pocos.

El concepto de agresión no excluye actos físicos hostiles, pero generalmente se debe hacer referencia a actos intencionales menos extremos que pueden causar daños físicos o psicológicos a otros, dentro de estos se encuentran golpes, 
empujones, aislamiento a grupos de iguales sin propósito, sobrenombres, todos constituyen ejemplos de actos agresivos. Estos pueden ser peligrosos, pero sus efectos generalmente son minimizados en contraste con los que están asociados a la hostilidad física.

Una subcategoría de la agresión es el denominado "Bullying" (Orpinas; Horne, 2006), cuyo término es difícil de traducir al español, pero que tiene una serie de connotaciones propias. Este término contempla a los niños y niñas que sufren de actos agresivos como es la intimidación, que es intencional y repetitiva a lo largo del tiempo; se añade a lo anterior que estos actos quedan excluidos del marco legal $y$ no son tipificados como tal.

Existe la tendencia a minimizar estos actos, $y$ verlos como una lucha y no como actos de intimidación $y$, por lo tanto, de victimización. Los niños y niñas pueden aprender que la agresión es una conducta aceptable, que forma parte de la intimidad y que esa agresión o miedo es una forma correcta de ejercer control sobre los demás, más aun conductas que se pueden considerar deseables. Esto lleva a los niños y niñas a conductas de introversión y pasividad, o a conductas disruptivas y agresivas hacia las cosas o las personas.

El ambiente familiar influye directamente sobre la autopercepción y la vulnerabilidad para sufrir agresión o perpetrar agresión que tienen los niños y las niñas, aun cuando ellos y ellas no sean agredidos en los episodios de agresión familiar, pero son observadores aterrados de situaciones de malos tratos, generalmente perpetrados por el padre o la madre. Aunque en un principio compadecen a la madre, conforme crecen esta solidaridad se transforma en hostilidad.

Cuando los niños y niñas ingresan a la escuela, su mundo familiar empieza a ser postergado para incorporarse a otro medio social; aparece su deseo y su temor de ser aceptado dentro de este nuevo grupo social. El conflicto que se presenta, más o menos importante, puede irse resolviendo según su temperamento e historia personal. Así optará por esforzarse en prevalecer sobre otros, se someterá a él, o bien se aislará.

La dinámica del grupo está determinada en gran medida por la estructura de las interacciones $y$ de las normas de comunicación que se establecen. El propio grupo genera y mantiene una estructura relacional especial, que en la gran mayoría de las veces no está bajo el control del docente.

Dentro de la dinámica de grupo en la escuela se encuentra una estructura informal de agresión: los "bullies" o agresores, las víctimas, $y$ los seguidores u observadores. Muchas de las conductas indeseadas de los "bullies" se adquieren $y$ se mantienen en gran parte por el refuerzo de los compañeros o compañeras. El grupo refuerza las conductas agresivas, ya que se valora más positivamente a los agresores que a las víctimas, lo cual se traduce por un lado, un mayor sentimiento de afiliación o temor a denunciar a los agresores y por otro, a que las víctimas se sienten más apartadas.

\section{PROCEDIMIENTO METODOLÓGICO}

El estudio realizado es descriptivo. Lo que se pretendía es describir como perciben los niños y niñas de preparatoria y primer ciclo la agresión. Danhke (1989) menciona que los estudios descriptivos buscan especificar las propiedades importantes de personas, grupos, comunidades o cualquier otro fenómeno que sea sometido a análisis.

El objetivo básico del estudio fue analizar el entorno inmediato de los niños y niñas en el ámbito de la agresión, cuya tesis central parte de la teoría sociocultural, $y$ consiste en que el desarrollo humano es el resultado de la interacción entre el individuo y la cultura. Dicho en otras palabras, el desarrollo del conocimiento y de las competencias individuales tienen lugar a través de la orientación, el apoyo y la estructura que proporciona el contexto socio-cultural (Vigotsky, 1978).

La población objeto fueron niños y niñas que cursaban preparatoria, primero, segundo y tercer grado. Las edades oscilaban entre los $5 y$ 9 años de edad. Se eligió estos grupos etarios, ya que, según Kohlberg (1969), en estas edades se da el establecimiento de los conceptos de moral (se considera como una fase primitiva), el juicio $y$ la sensibilidad moral basado en el respeto de la norma y la autoridad que emana del grupo, de 
tal manera, que los juicios y decisiones poseen un trasfondo de utilidad (cooperación y bienestar social) pero que al mismo tiempo, resulta de interiorizar reglas socialmente enunciadas. Además puede dar pie a la implementación de programas de prevención en el ambiente escolar.

Se eligieron 154 niños y niñas de cuatro escuelas del área metropolitana, en las cuales se encontraban trabajando estudiantes de la Escuela de Enfermería de la Universidad de Costa Rica, los cuales recolectaron la información a través de un cuestionario con preguntas abiertas, estructuradas en tres áreas importantes: primero el área sociodemográfica, que posibilitaba caracterizar a la población en estudio; una segunda área escolar, que brindaba información sobre el comportamiento de situaciones de agresión en la escuela; y por último, el área familiar. Además la investigadora realizó una observación no participante de cuatro meses en una de las escuelas seleccionadas, dos días por semana.

El análisis de los datos planteado fue estadístico univariable, con el paquete estadístico del SPSS, realizándose un análisis exhaustivo de cada variable incluida en la matriz de datos, obteniéndose las frecuencias relativas o porcentuales y acumuladas, proporcionándose la cantidad de casos válidos. La presentación se hizo a través de cuadros en frecuencias absolutas y porcentajes.

Se analizaron varias áreas importantes a saber:

1. El área socio demográfica

2. Conocimiento sobre agresión

3. El área escolar

4. El área familiar

\section{PRESENTACIÓN Y ANÁLISIS DE LOS DATOS}

Primero se iniciará con una caracterización de la población, que se presenta en el cuadro 1. Como se observa un poco más de la mitad $(51,2 \%)$ de la población está constituida por varones y el $48,7 \%$ son niñas, con una distribución porcentual mayor en el grupo etario de los 7 años.

\section{CUADRO 1}

DISTRIBUCIÓN DE LOS NIÑOS Y NIÑAS SEGÚN EDAD Y SEXO EN NÚMEROS PORCENTUALES Y RELATIVOS. COSTA RICA, 2005

\begin{tabular}{|c|c|c|c|c|c|c|}
\hline \multirow[t]{3}{*}{ EDAD } & \multicolumn{4}{|c|}{ SEXO } & \multirow{2}{*}{\multicolumn{2}{|c|}{ TOTAL }} \\
\hline & \multicolumn{2}{|c|}{ FEMENINO } & \multicolumn{2}{|c|}{ MASCULINO } & & \\
\hline & $\mathrm{N}$ & $\%$ & $\mathrm{~N}$ & $\%$ & $\mathrm{~N}$ & $\%$ \\
\hline 5 años & 13 & 17,3 & 6 & 7,6 & 19 & 12,3 \\
\hline 6 años & 16 & 21,3 & 13 & 16,5 & 29 & 18,8 \\
\hline 7 años & 38 & 50,7 & 37 & 46,8 & 75 & 48,7 \\
\hline 8 años & 20 & 10,7 & 20 & 25,3 & 28 & 18,2 \\
\hline 9 años & & & 3 & 3,8 & 3 & 1,9 \\
\hline TOTAL & 75 & 100,0 & 79 & 100,0 & 154 & 100,0 \\
\hline
\end{tabular}

Fuente: Leiva, V. "Percepción de agresividad en un grupo de niños y niñas de Kinder y primer ciclo de Educación General Básica”. Escuela de Enfermería, Universidad de Costa Rica.

El 66,9\% de los niños y niñas ocupan el primero o segundo periodo ínter genésico entre sus hermanos y hermanas, es decir, que son los hijos mayores o segundos de la familia. Así mismo el
$67,5 \%$ viven con ambos padres, familias nucleares y el $27,9 \%$ viven solo con su madre.

A continuación se ofrece los resultados por áreas determinadas en el apartado anterior. 


\section{CONOCIMIENTO SOBRE AGRESIÓN}

Era de interés conocer si los niños y niñas conocían el término de agresión y se encontró que 70,8\% conocían el significado de la palabra agresión, siendo tan solo ligeramente discreta la diferencia (3\%) entre hombres $y$ mujeres, con predominio mayormente positivo en la edad de los 7 años con un 56,9\%.

De los que contestaron afirmativamente conocer la palabra agresión, entre los sitios más frecuentes en que han oído la palabra agresión se encuentran en primer lugar los programas de televisión $37,6 \%$, tanto en programas infantiles como en no aptos para menores pero que sus tutores permiten o creen que no tiene nada de "malo"; seguida en la escuela con un 15,6\% y la casa con un $8,4 \%$. Esto también se mostró en relación con la edad.

Un $71,4 \%$ de las y los encuestados asociaron la agresión a un aspecto físico $y$ verbal, con predominio del primero $(56,5 \%)$, dentro de estos se encuentran: golpes, patadas y palabras groseras en la escuela, castigo físico que va desde jalones de pelo, pellizcos o fajazos de los padres, entre los más importantes. Muy pocos niños y niñas lo asociaron a agresión psicológica, sexual, o por descuido $(10,4 \%)$.

\section{EL ÁREA ESCOLAR}

Fue de interés analizar que pasaba sobre las situaciones de agresión en el medio escolar. Como se muestra en el cuadro 2 , un $63,3 \%$ de los niños y niñas refieren que en su grupo de clase se presenta agresión. En cuanto a los grupos etarios, aunque en todos las edades estudiadas describen acciones violentas, el mayor porcentaje se obtuvo en el grupo etario de los 7 años $(29,2 \%)$.

\section{CUADRO 2}

\section{DISTRIBUCIÓN DE LOS NIÑOS Y NIÑAS DEL GRUPO DE ESCUELA} SEGÚN SEÑALA LA PRESENCIA DE AGRESIÓN

\begin{tabular}{|c|c|c|c|c|c|c|}
\hline \multirow[t]{3}{*}{ SEXO } & \multicolumn{6}{|c|}{ PRESENCIA DE AGRESIÓN EN EL GRUPO DE CLASE } \\
\hline & \multicolumn{2}{|c|}{ SI } & \multicolumn{2}{|c|}{ NO } & \multicolumn{2}{|c|}{ NO RESPONDE } \\
\hline & $\mathrm{N}$ & $\%$ & $\mathrm{~N}$ & $\%$ & $\mathrm{~N}$ & $\%$ \\
\hline FEMENINO & 50 & 51,6 & 16 & 41,0 & 10 & 52,6 \\
\hline MASCULINO & 46 & 48,4 & 23 & 59,0 & 9 & 47,4 \\
\hline TOTAL & 96 & 100,0 & 39 & 100,0 & 19 & 100,0 \\
\hline
\end{tabular}

Fuente: Leiva, V. "Percepción de agresividad en un grupo de niños y niñas de Kinder y primer ciclo de Educación General Básica”. Escuela de Enfermería, Universidad de Costa Rica.

Se indagó sobre cual era la víctima de las acciones violentas dentro del aula y se encontró que la agresión era ejercida en primera instancia contra un compañero o compañera (53,9\%), o bien contra todo el grupo o contra el o la niña entrevistada en una misma proporción (5,8\%):

—Sí, Alonso, nada más él, nos golpea a mí, a Marcos y Carlos, yo me siento mal, después yo lo golpeo un poquito y la maestra nos castiga poniéndonos a hacer fila.
Cabe destacar que como se muestra en el cuadro anterior, 58 niños y niñas respondieron negativamente o se abstuvieron de manifestar si en su grupo había agresión, pero al pedírseles que dieran un ejemplo de las cosas violentas que hacían, estos unidos al resto de los encuestados refirieron acciones violentas en el orden físico y verbal $(63,6 \%)$, dentro de estas se encontraron "golpes, empujones, pellizcos, jalar el pelo, patear, palabras ofensivas alusivas a características físicas, sexo, procedencia, o 
eficiencia académica”. Una niña refirió acciones relacionadas con la agresión sexual de parte de un compañero con discapacidad, que acosaba a las niñas de su grupo, sin embargo, dichas acciones no fueron valoradas en orden de agresión sexual por parte del resto del grupo.

En cuanto a las reacciones de los niños y las niñas que respondieron afirmativamente la presencia de acciones de agresión en su grupo, el $54,5 \%$ de ellos y ellas refirieron sentirse mal, $3,9 \%$ enojados y un 2,6\% bien (cuadro 3). Asociado a lo anterior cuando suceden situaciones violentas dentro de su escuela, un $48 \%$ de ellos y ellas buscan a un adulto (en todos esos casos fue la maestra) mientras que otros optan por alejarse $(28,6 \%)$, no hacer nada $(18,4 \%)$ o responden con agresión $(12,3 \%)$, siendo estos en su totalidad varones.

CUADRO 3

DISTRIBUCIÓN POR SEXO SEGÚN RESPUESTA ANTE ACCIONES VIOLENTAS EN SU GRUPO. COSTA RICA, 2005

\begin{tabular}{|c|c|c|c|c|c|c|c|c|c|c|}
\hline \multirow[t]{3}{*}{ SEXO } & \multicolumn{8}{|c|}{ QUE HACES } & \multirow{2}{*}{\multicolumn{2}{|c|}{ TOTAL }} \\
\hline & \multicolumn{2}{|c|}{ NADA } & \multicolumn{2}{|c|}{ ME ALEJO } & \multicolumn{2}{|c|}{$\begin{array}{c}\text { BUSCO A UN } \\
\text { ADULTO }\end{array}$} & \multicolumn{2}{|c|}{$\begin{array}{c}\text { RESPONDO CON } \\
\text { AGRESIÓN }\end{array}$} & & \\
\hline & $\mathrm{N}$ & $\%$ & $\mathrm{~N}$ & $\%$ & $\mathrm{~N}$ & $\%$ & $\mathrm{~N}$ & $\%$ & $\mathrm{~N}$ & $\%$ \\
\hline Femenino & 12 & 66,6 & 10 & 47,6 & 29 & 61,7 & 0 & 0 & 51 & 52,1 \\
\hline Masculino & 6 & 33,4 & 11 & 52,4 & 18 & 38,3 & 12 & 100,0 & 47 & 47,9 \\
\hline TOTAL & 18 & 100,0 & 21 & 100,0 & 47 & 100,0 & 12 & 100,0 & 98 & 100,0 \\
\hline
\end{tabular}

Fuente: Leiva, V. "Percepción de agresividad en un grupo de niños y niñas de Kinder y primer ciclo de Educación General Básica”. Escuela de Enfermería, Universidad de Costa Rica.

Fue de interés conocer que hacen los compañeros y compañeras de los entrevistados cuando suceden acciones violentas en su grupo de escuela, estos refieren que ellos y ellas no hacen nada (37\%), acuden a un adulto (35,8\%), o responden agrediendo $(27,2 \%)$ al niño o niña que está ejecutando acciones violentas.

Las acciones del adulto que llega en auxilio de los niños y niñas que están siendo víctimas se resumen en el cuadro 4. Los niños y niñas refieren que la medida más importante que utiliza la maestra es enviar mensajes al hogar $(58,6 \%)$ del que está realizando acciones violentas, seguida por corrección verbal, es decir regañar $(39,1 \%)$; tan solo dos niños o niñas refirieron que su maestra gritaba o no hacía nada ante estas situaciones.

\section{CUADRO 4}

\section{DISTRIBUCIÓN POR SEXO, SEGÚN RESPUESTA DE LA MAESTRA ANTE ACCIONES VIOLENTAS. COSTA RICA, 2005}

\begin{tabular}{|c|c|c|c|c|c|c|c|c|c|c|}
\hline \multirow[t]{3}{*}{ SEXO } & \multicolumn{8}{|c|}{ QUÉ HACE LA MAESTRA } & \multirow{2}{*}{\multicolumn{2}{|c|}{ TOTAL }} \\
\hline & \multicolumn{2}{|c|}{ REGAÑA } & \multicolumn{2}{|c|}{$\begin{array}{c}\text { ENVÍA MENSAJES } \\
\text { AL HOGAR } \\
\end{array}$} & \multicolumn{2}{|c|}{ GRITA } & \multicolumn{2}{|c|}{ NADA } & & \\
\hline & NRO & $\%$ & NRO & $\%$ & NRO & $\%$ & NRO & $\%$ & NRO & $\%$ \\
\hline Femenino & 14 & 41,2 & 29 & 56,8 & 1 & 100,0 & 1 & 100,0 & 45 & 51,7 \\
\hline Masculino & 20 & 58,8 & 22 & 43,2 & & & & & 42 & 48,3 \\
\hline TOTAL & 34 & 100,0 & 51 & 100,0 & 1 & 100,0 & 1 & 100,0 & 87 & 100,0 \\
\hline
\end{tabular}

Fuente: Leiva, V. "Percepción de agresividad en un grupo de niños y niñas de Kinder y primer ciclo de Educación General Básica”. Escuela de Enfermería, Universidad de Costa Rica 


\section{EL ÁREA FAMILIAR}

Fue de interés conocer si los niños y niñas encuestados percibían situaciones violentas dentro de sus hogares, encontrándose que un $63,3 \%$, refieren no tener acciones violentas en sus casas, $y$ un $36,7 \%$ sí refieren acciones violentas (cuadro 5). Siendo los varones quienes refirieron menos situaciones de agresión en sus casas.

\section{CUADRO 5}

\section{DISTRIBUCIÓN POR SEXO SEGÚN ACCIONES VIOLENTAS EN \\ LA CASA. COSTA RICA, 2005}

\begin{tabular}{ccccc}
\hline \multirow{2}{*}{ SEXO } & \multicolumn{3}{c}{ ACCIONES VIOLENTAS EN LA CASA } \\
\cline { 2 - 5 } & \multicolumn{3}{c}{ SI } & \multicolumn{3}{c}{ NO } \\
\cline { 2 - 5 } Femenino & $\mathrm{N}$ & $\%$ & $\mathrm{~N}$ & $\%$ \\
\cline { 2 - 5 } Masculino & 30 & 53,6 & 44 & 44,9 \\
\hline TOTAL & 56 & 46,4 & 54 & 55,1 \\
\hline
\end{tabular}

Fuente: Leiva, V. "Percepción de agresividad en un grupo de niños y niñas de Kinder y primer ciclo de Educación General Básica”. Escuela de Enfermería, Universidad de Costa Rica.

Según la edad, los grupos que refirieron que en su casa se daban situaciones violentas se encuentra en primer lugar los niños de 6 años con un $48,2 \%$ para el número total de su grupo, seguido por los niños y niñas de 8 años con un $35,7 \%$, $y$ en tercer lugar los niños y niñas de 7 años con un $26,6 \%$.
Asimismo, las y los encuestados que respondieron afirmativamente sobre situaciones violentas, refirieron que un $46,5 \%$ esas situaciones eran escenificadas por ambos padres, un $44,8 \%$ por sus hermanos o hermanas $y$ un $8,6 \%$ por otras personas, dentro de las que se incluyen tíos, tías o primo.

En el cuadro 6 se ofrece las principales acciones violentas que el niño o niña presencia en sus hogares. Como se puede observar el 58,6\% de los niños y niñas manifestaron que el tipo de agresión predominante en sus casas es el físico, seguido por el verbal y el físico-verbal con un $18,9 \%$ respectivamente, la gran mayoría es efectuada por los adultos o hermanos $y$ hermanas mayores sobre los niños y niñas más pequeños. En el caso de los padres y madres, muchas de las acciones violentas están en el orden correctivo o disciplinario: jalar el pelo, pegar con faja, pegar con la mano, pellizcar, entre los más frecuentes; en el orden verbal, se hace alusión a la comparación entre hermanos, su capacidad académica, entre los más mencionados. La agresión sexual se presentó en dos niños, que cabe aclarar no eran ellos las víctimas sino sus madres, siendo los victimarios en un caso, el compañero afectivo de una y en el otro, el hijo. Asimismo, mucho de lo descrito por los niños y niñas encuestados está relacionado a conflictos de pareja importantes, ya sea entre sus padres o con un o una compañera afectiva de alguno de ellos.

En cuanto a como se sienten ante esas situaciones de agresión, el 56,9\% refirieron sentirse mal, el 12,1\% asustado(a) o triste, el 6,9\% enojada, enojado o bien.

\section{CUADRO 6}

\section{DISTRIBUCIÓN POR SEXO SEGÚN TIPO DE AGRESIÓN EN EL HOGAR COSTA RICA, 2005}

\begin{tabular}{|c|c|c|c|c|c|c|c|c|}
\hline \multirow[t]{3}{*}{ SEXO } & \multicolumn{8}{|c|}{ TIPO DE AGRESIÓN EN EL HOGAR } \\
\hline & \multicolumn{2}{|c|}{ FÍSICA } & \multicolumn{2}{|c|}{ VERBAL } & \multicolumn{2}{|c|}{ FÍSICA Y VERBAL } & \multicolumn{2}{|c|}{ SEXUAL } \\
\hline & $\mathrm{N}$ & $\%$ & $\mathrm{~N}$ & $\%$ & $\mathrm{~N}$ & $\%$ & $\mathrm{~N}$ & $\%$ \\
\hline Femenino & 24 & 70,6 & 6 & 54,5 & 2 & 18,2 & 0 & 0 \\
\hline Masculino & 10 & 29,4 & 5 & 45,5 & 9 & 81,8 & 2 & 100,0 \\
\hline TOTAL & 34 & 100,0 & 11 & 100,0 & 11 & 100,0 & 2 & 100,0 \\
\hline
\end{tabular}

Fuente: Leiva, V. "Percepción de agresividad en un grupo de niños y niñas de Kinder y primer ciclo de Educación General Básica”. Escuela de Enfermería, Universidad de Costa Rica. 
En cuanto a qué hace el niño o la niña cuando hay acciones violentas en su casa, se encontró que ellos y ellas tienen medidas como establecer distancia de la situación violenta, así un 39,6\% se alejan o no hacen nada, un $15,5 \%$ buscan ayuda, un $13,7 \%$ tan solo llora $y$ un $20,7 \%$ responde agresivo o interviene, esto se da en la mayoría de los casos cuando están golpeando a la madre. Algunas de las cosas narradas por los niños $y$ niñas, es que ellos $y$ ellas muchas veces tienen que tratar de intervenir para detener la situación que se está dando en su casa.

En cuanto a los hermanos o hermanas de los niños y niñas encuestados poseen un comportamiento similar al comportamiento de estos. Cabe aclarar que muchos de los niños y niñas poseen hermanos muy pequeños, o no viven ya en su casa o son hijos únicos, por lo que la no respuesta en este rubro fue alta (13,2\%).

En cuanto a las medidas realizadas por los adultos para poder resolver las acciones violentas dentro de la casa son con medidas agresivas, como pegar (27,6\%), gritar (15,5\%), regañar $(8,6 \%)$ o hablar $(12,1 \%)$, mientras que un $15,5 \%$ no intervienen. Hay que tomar en cuenta en esta parte que en la gran mayoría los víctimarios son ambos padres y las acciones agresivas se dirigen entre ellos, en disputas de pareja, contra uno o una de las hijas o contra el niño o la niña encuestada.

\section{DISCUSIÓN DE LOS DATOS}

Para iniciar es importante decir que los niños y niñas conocen la palabra agresión o violencia. Como definición ellos y ellas lograron identificarla claramente, asimismo el sitio en el que estos observan acciones violentas es la televisión, $y$ esto ha sido estudiado exhaustivamente, no solo por la preocupación social sobre el exceso de escenas de violencia presentes en la televisión, sino también, por las repercusiones que las imágenes violentas presentes en los programas ejercen sobre el público infantil (Aran, Barata, Busquet, y otros, 2002, 2003) (Fiske, 1987). Agregado a esto, la escuela y la casa, Bandura y Walters (1963), Berkowitz (1996) y Friedrich y Huston (1986), sostienen desde la psicología conductual que la televisión, así como las interacciones sociales con otras personas ya sea en el hogar, o en la escuela, como los principales agentes socializadores, pueden ser fuentes transmisoras de comportamientos violentos que propician un aprendizaje por modelaje y condicionado de pautas agresivas, convirtiéndose dentro de la teoría del efecto causal entre la visión de la violencia o agresión en la televisión, en la escuela o el hogar y las conductas agresivas que pueden mostrar los niños y niñas.

Se encontró que en el ambiente escolar se da una serie de actos agresivos, entre compañeras y compañeros, pero que no compromete a las maestras, sino que queda al nivel de relaciones entre el grupo de iguales. La mayoría de las acciones agresivas se dan en el espacio en que no hay supervisión de adulto, como son los recreos, o las actividades libres.

Estas acciones caen dentro del término "bullying" (Orpinas, Horne, 2006), ya que va desde la agresión física con el uso intencional de la fuerza física, la agresión verbal con el uso intencional de palabras que causan daño emocional y psicológico, $y$ en menor frecuencia, pero no menos importante, la agresión relacional con conductas que hacen que algún niño o niña sea apartado, rechazado o aislado.

Es importante analizar que cuando suceden actos agresivos dentro de los grupos, los niños y niñas buscan ayuda en primera instancia en un adulto, en estos casos se trata de la maestra, la cual utiliza como medida correctiva primordialmente el elaborar una nota en el cuaderno de mensaje a los padres sobre la conducta del niño o la niña. Es decir, deposita en los padres la responsabilidad de corregir las conductas de sus hijos e hijas, en espacios que son dominios de la maestra, desautorizándose a sí misma como adulto responsable. No se toma en cuenta si esos padres saben o no corregir de una manera positiva estas conductas agresivas.

Asímismo, los niños y niñas adoptan una posición, en la cual se visualiza claramente tres actores, mencionados por Orpinas, Horne, 2006:

$\diamond$ El bullyes o víctimario: es el niño o niña que ejerce intimidación, agresión abierta física o de palabra. 
४ La víctima: que el término más apropiado debería ser "blanco de la agresión" ya que la víctima denota impotencia, que en la gran mayoría de los casos no se da por diferencias físicas importantes. En la narrativa de los niños y niñas se puede distinguir el blanco de agresión pasivo, que es aquel niño o niña que no hace nada para provocar, caracterizado por ser un niño o niña tímido, con pocos amigos, sin muchas habilidades sociales. El blanco de la agresión provocador, es decir, hacen cosas o dicen palabras que saben van a causar conductas agresivas hacia sí mismo. También los niños y niñas blanco de agresión relacional, son aquellos que son víctimas porque son más inteligentes, o se destacan del resto del grupo por poseer habilidades o destrezas en áreas en que el resto no se destacan.

$\diamond$ Y por último, los espectadores: los compañeros y las compañeras que no son blanco de la agresión, que ven lo que sucede, estos se dividieron según el estudio. Primero, en aquellos $y$ aquellas que son parte del problema en los cuales se encuentran los y las que incitan al niño o niña a perpetrar actos agresivos, o que tratan de detener la agresión por medio de agresión. Y los niños y niñas que son parte de la solución, aquellos que buscan ayuda, tratan de intervenir, apoyando al niño o niña blanco de la agresión, tratando de separarlos.

En el ambiente familiar no fue menos importante la información recopilada que nos lleva a la siguiente discusión.

Los padres recogen la voluntad de la cultura dominante para que se encarguen de disuadir cualquier intento que contraríe el orden social establecido e incluso, aceptan un programa de castigo-sanción, que inclusive no es admitido como tal, sino como un programa de disciplina por el bien de los hijos y del hogar.

Independientemente de cual sea el porcentaje de familias comprometidas en la violencia, no se puede negar que la familia es la institución social en donde el ejercicio de la violencia se practicó con mayor frecuencia e intensidad que en otros escenarios sociales (Corozzo,1999; Arnicama, 1999 citados por Campos, 1999).
Esto es evidente en lo expuesto por los y las entrevistadas, en que los ejemplos de agresión en sus casas está asociados a medidas "correctivas" de los padres, hacia ellos o sus hermanos, así como violencia física y verbal entre los padres. Llama la atención que los niños y niñas refieren disputas con sus hermanos o hermanas o entre ellos, que corresponden al orden de rivalidad fraterna $y$ los padres en su mayoría utilizan la agresión, para poner fin, a las disputas entre los hermanos. El castigo físico, como medida disciplinaria, queda circunscrito al orden privado y es socialmente avalado (Roth, 1997) . Dentro de este se emplea la fuerza física para causar dolor, sin lesionar, con el propósito de corregir o controlar conductas "inadecuadas"; entre las mencionadas con mayor frecuencia por los niños y niñas se encuentran: pegar con la mano, pegar con la faja, pellizcar, tirar del cabello.

Para finalizar, es importante retomar que en la mayoría de las niñas y los niños encuestados refieren sentirse mal por las acciones violentas que viven en la escuela y en su casa, sin embargo, ellos perciben que es algo contra lo que no se puede luchar, sino que es asumido como algo cotidiano $y$ que hay que acostumbrarse. Asimismo, este sentimiento es reforzado por la conducta de impotencia o inatención que presentan los adultos encargados, cuyo mensaje lleva implícito una aprobación hacia la violencia como forma de solución de conflictos sociales.

\section{CONSIDERACIONES FINALES}

En este apartado se ofrece un resumen breve de las consideraciones más importantes de la investigación:

$\diamond$ La violencia estructural que se da en todos los ámbitos sociales en nuestro país se filtra en nuestros estudiantes, a través de la agresión escolar.

$\diamond$ Los niños y niñas están expuestos durante largas horas del día a la violencia omnipresente en los medios de comunicación social, principalmente la televisión. Sin embargo, cabe decir que no se debe sobre dimensionar estos medios, ya que los niños y las niñas 
poseen otras instancias sociales y culturales, como la escuela o la familia, que siguen teniendo un peso considerable y gran responsabilidad social en la educación infantil. Pero cabe preguntar ¿están cumpliendo a cabalidad estas instancias con la responsabilidad que la sociedad les ha encomendado? O ¿será que es precisamente el papel que se espera de la escuela y la familia como instancias reforzadoras de una sociedad básicamente violenta?

$\diamond$ Se encontró que el "bullying" es un fenómeno oculto o manifiesto comportamiento antisocial, dentro de las escuelas.

$\diamond$ Los niños y niñas con los que se trabajó, son de edades relativamente pequeñas, y se manifiestan actos agresivos de importancia, que llevan a un aprendizaje por modelaje de solución de conflictos sociales a través de la agresión.

$\diamond$ Por tanto, es importante que los programas dirigidos a la prevención de la violencia, deben ser desarrollados en estos grupos, pues se ha demostrado que a temprana edad, ya los niños y niñas instauran las conductas agresivas como formas relacionales.

$\diamond$ Las maestras y maestros ponen mayor énfasis en atender los comportamientos disruptivos en el aula, o relacionales profesor alumno o alumna, no así al maltrato entre compañeros o compañeras (bullying).

$\diamond$ Se pone especial importancia a actos agresivos, que se consideran lesionantes como la agresión física moderada o severa, que puede conllevar a incapacidad o la muerte y a la agresión sexual. Pero no así a la intimidación, la ofensa, el aislamiento, el acoso, ya que son considerados parte de las relaciones entre grupos de pares, pero que causan daños irreversibles: abandono escolar, suicidio $y$ homicidio.

$\diamond$ La escuela es una de las principales venas del desarrollo social, en la cual los niños y niñas establecen relaciones interpersonales, forzados a una convivencia cercana, donde ser aceptado por sus iguales es de vital importancia. Los educadores consejeros, deben de buscar formas positivas de relación entre los niños y niñas, de manera que ellos y ellas posean herramientas positivas para solventar conflictos a través de la comunicación y no la agresión o la victimización (Seligman y otros, 1995).

$\diamond$ En el seno familiar se perpetúan las acciones violentas, que se distinguen claramente en dos vías, la primera hacia los niños y niñas en el orden correctivo o disciplinario, y la segunda como forma de resolución de conflicto de relación principalmente de pareja de los progenitores.

$\diamond \mathrm{Al}$ igual que en la escuela, en el hogar se refuerza el sentimiento de impotencia en los niños y niñas, ante los actos de agresión, por lo cual, no es de extrañar que se instaure, no solo la habituación a la violencia, sino el sentido de que no se puede hacer nada contra la violencia en la vida adulta.

\section{BIBLIOGRAFÍA}

Aran, S.; Barata, F.; Busquet, J.; Medina, P.; Moron, S. "Childhood, Violence and Television. Use and childhood perception of violence in Televisión". Violénce $i$ mitjans de comunicació: recursos $i$ discursos. Barcelona: Tripodos, 2003.

Aran,S.; Barata, F.; Busquet, J.; Medina, P. La violéncia en la mirada. L' análisi de la violencia a la televisió. Barcelona: Papers d' estudi, 2002.

Bandura, A.; Walters, R. Social learning and personality development. New York: Holt, Rinehart and Winston, 1963.

Berkowitz, L. Agresión causa, consecuencias y control. Bilbao: Declée de Brouwer, 1996.

Campos, J. "La violencia psicológica". Revista Peruana de Psicología 5. Lima, 50-57.

Danhke, G. "Investigación y comunicación". Fernández-Collado, C. y Danhke, G. (comps). La comunicación humana: 
ciencia social. México: McGraw-Hill, 1989

Fiske, J. Television culture. Londres: Routledge, 1987

Friedrich, L.; Huston, A. "Television violence and aggression: The debate continues". Psychological Bulletin 100. Washington, 1986: 364-371.

Kohlberg, L. Stages in the development of moral thought and action. New York: Holt, 1969.

Krug, E.; Shaffer, A.; Dorsey, S. and Forehand, R. "The world report on violence and health". Lancet 360. 2004: 1038-1088.

Orpinas, P.; Horne, A. Bulling prevention: Creating a positive school climate and developing social competence. Washington: American Psychological Association, 2006.

Roth, C. Informe Anual sobre el respeto de los derechos humanos en la Unión Europea. (s.a): Comisión de Libertades Públicas $y$ de Asuntos Interiores. Parlamento Europeo, 1997.

Seligman, M.; Reivich, K.; Jaycox , L. and Gillham, J. The optimistic child: A proven program to safeguard children against depression and build lifelong resiliency. New York: Harper Perennial, 1995.

Vigotsky, L. Mind in society: The development of higher psychological processes. Cambridge: Harvard University Press, 1978. 
